rev.relac.int.estrateg.segur.8(2):119-142,2013

\title{
DE LOS CONFLICTOS COMPLEJOS: NATURALEZA, ESTRUCTURA Y MORFOLOGÍA DE LOS CONFLICTOS INTRATABLES E IMPERECEDEROS*
}

\author{
Vicente Torrijos Rivera** \\ Andrés Pérez Carvajal ${ }^{* * *}$
}

\section{RESUMEN}

En el presente artículo se aborda la naturaleza de los conflictos, sus principales componentes estructurales y su composición morfológica, desde el cambio de perspectiva (no lineal, ni determinista) propuesto por las teorías de la complejidad para las ciencias sociales. El análisis se centra en el estudio de los conflictos intratables, como fenómeno en el que interactúan de forma dinámica e interdependiente múltiples variables, creando un sistema complejo al que es necesario abordar como un todo y no por sus componentes particulares.

* Proyecto de investigación: “Tendencias evolutivas del terrorismo en Colombia / las FARC, 2010 - 2019", Línea de investigación: "Paz, Seguridad y Conflictos" (denominación vigente a enero del 2013) número de identificación del proyecto es: 0025 289. Financiado por el Centro de Estudios Políticos e internacionales, Universidad del Rosario. Clasificación (Journal of Economic Literature): D740 Conflictos; resolución de conflictos; Alianzas.

** Profesor Titular y Distinguido de Ciencia Política y Relaciones Internacionales en la Universidad del Rosario, doctorado en Relaciones Internacionales y el postdoctorado en asuntos estratégicos, seguridad y defensa. Bogotá, Colombia. Comentarios a: tutoriascontorrijos@yahoo.com

*** Asistente de Investigación del proyecto "Tendencias evolutivas del terrorismo en Colombia / las FARC, 2010 - 2019", Graduando en la Facultad de Ciencia Política y Gobierno, y becario en el programa de Historia de la Universidad del Rosario, Bogotá, Colombia. Comentarios a: perez.andres@urosario.edu.co 
El objetivo es estudiar la composición morfología que hace a este tipo de conflictos particularmente violentos, perdurables y resistentes a la trasformación pacífica. En función de esto, se expone la forma en que las estructuras psicosociales y la identidad grupal se convierten en los factores determinantes en la formación y composición que adquiere un conflicto intratable. Adicionalmente, se propone complejizar el entendimiento del tiempo como variables central de la intratabilidad, explicando las lógicas que permiten la estabilización de conflictos y el transito generacional por medio de las ideas de institucionalización de estructuras psicosociales y los atractores de conflicto.

Palabras Clave: Conflictos, Conflictos intratables, atractores e institucionalización de conflictos.

\title{
COMPLEX CONFLICTS: NATURE, STRUCTURE AND MORPHOLOGY OF INTRACTABLE AND ENDURING CONFLICTS
}

\begin{abstract}
This document analyzes the nature of conflicts, its main structural components and its morphological composition, from the change of perspective (not lineal nor deterministic) proposed by the complexity theories for social sciences. This analysis focuses on the study of intractable conflicts, as a phenomenon in which multiple variables interact dynamically and interdependent of each other, creating a complex system which needs to be approached as a whole and not by its individual components.

The objective is to study the morphological composition that makes this type of conflicts uniquely violent, enduring and resistant to a pacific resolution. Based on this, the psychological structure and group identity become the decisive factors on the molding and composition that an intractable conflict acquires. Additionally, it is proposed to increase the complexity of the understanding of "time" as a central variable of intractable conflicts, while explaining the logic that permits the normalization of conflicts and generational transition, using the institutionalization of psychological structures and conflict attractors.
\end{abstract}

Keywords: Conflicts, intractable conflicts, attractors, institutionalization of conflicts.

\section{SOBRE OS CONFLITOS COMPLEXOS: NATUREZA, ESTRUTURA E MORFOLOGIA DOS CONFLITOS INTRATÁVEIS E IMPERECÍVEIS}

\section{RESUMO}

Este artigo aborda a natureza dos conflitos, seus principais componentes estruturais e a sua composição morfológica, a partir de uma mudança da perspectiva (não linear ou determinista) 
proposta pelas teorias da complexidade das ciências sociais. A análise centra-se no estudo dos conflitos intratáveis como um fenômeno no qual interagem de maneira dinâmica e interdependentes múltiplas variáveis, criando um sistema complexo que precisa ser bordado como um todo e não somente pelos seus componentes individuais.

O objetivo é estudar a composição morfológica que torna este tipo de conflitos violentos, duradouros e resistentes à transformação pacífica. Em função do anterior, expõe-se como as estruturas psicossociais e a identidade grupal são fatores determinantes na formação e composição de um conflito, tornando-o intratável. Além disso, propõe-se um entendimento mais complexo do tempo como uma das variáveis centrais da intratabilidade, explicando a lógica que permite a estabilização dos conflitos e o trânsito geracional por meio das ideias de institucionalização de estruturas psicossociais e dos atratores de conflitos.

Palavras-chave: conflito, conflitos intratáveis, atratores e institucionalização de conflitos.

\section{INTRODUCCIÓN}

La paz, iniciando la segunda década del siglo XXI, continua siendo una de las mayores ambiciones inconclusas del espíritu y la inventiva humana. $Y$ es que ni el terror derivado de la era nuclear, ni las fantasías del progreso y la civilización de la modernidad, han logrado alejar a los humanos de la violencia y sus nefastas consecuencias (Joas, 2005:47-63).

Federico Aznar Fernández (2011:15), en su revisión de la naturaleza de la guerra, recuerda que aunque entre el siglo XVI y el XX la cantidad de guerras mayores ha descendido considerablemente, el número de muertes directas ocasionadas por la guerra se han elevado de forma radical, pasando de 15 millones de fatalidades en el siglo XIX, a las 187 millones de muertes, la mayoría de ellas de civiles o no combatientes, registradas en las guerras del siglo XX. Sin embargo, aunque nadie en su sano juicio podría considerar que la guerra y la violencia dejaran de ser parte de la historia de la humanidad en un tiempo considerable, el desaliento y el pesimismo no debe distraer a los académicos, analistas y políticos de continuar en la búsqueda por constituir un entorno global armónico, en el que sea posible la convivencia pacífica entre los hombres (Pizarro, 2005:29).

Bajo esta premisa es que se han perfilado los estudios sobre la paz y los conflictos, desde los que se reconoce que el estudio de los conflictos no significa de ninguna forma hacer apología a los mismos, por el contrario, se entiende como el paso inicial en la determinación de sus características fundamentales, y la búsqueda de los mecanismo que permitan su resolución pacífica y armónica (Fisas, 2002:17-37).

Es sobre esta perspectiva que se construyen las siguientes páginas, en las que se busca recomprender las lógicas de formación, desarrollo y perdurabilidad de los conflictos intratables. 
Esta manifestación extrema y duradera de la violencia, vivida en nuestros días a través del conflicto palestino israelí y las guerras étnico religiosas en África subsahariana, se ha convertido en la muestra más desalentadora, pero al mismo tiempo desafiante, de la necesidad de volver la atención sobre el análisis y la compresión de las dinámicas de los conflictos y la violencia, para la búsqueda de la paz.

A propósito de esto, el presente artículo propone tomar como epicentro de la intratabilidad una noción que aún no se trabajada dentro del campo: lo imperecedero. A través de esta categoría, se complejiza el entendimiento del tiempo, la identidad grupal dentro de los conflictos, los procesos de estabilización de conflictos y el transito generacional, como dimensiones fundamentales de la intratabilidad.

El objetivo de este modelo de análisis de la perdurabilidad de los conflictos es delimitar los espacios sobre los cuales los mediadores y facilitadores deben enfocar sus esfuerzos, proponiendo el dimensionamiento de las dinámicas conflictivas como paso insalvable en la posibilidad de transformar pacíficamente un conflicto intratable. En este sentido, el artículo no cae en la trampa, común en el campo académico, de formular metodologías de intervención y resolución que no atienden el análisis detallado y minucioso del tiempo como epicentro de la alta hostilidad y la resistencia al cambio.

En el artículo se abordan, en primer lugar, la teoría general de los conflictos, deteniéndose en sus principales elementos, procesos y configuración morfológica. En la segunda parte del escrito se describe la naturaleza, morfología y dinámica de interacción de los conflictos intratables. En la parte final, se propone la noción de proceso imperecedero como epicentro de las condiciones de retroalimentación y resistencia al cambio positivo, de la intratabilidad como característica definitoria de un conflicto. En el siguiente esquema se consigna la lógica de trabajo del presente artículo.

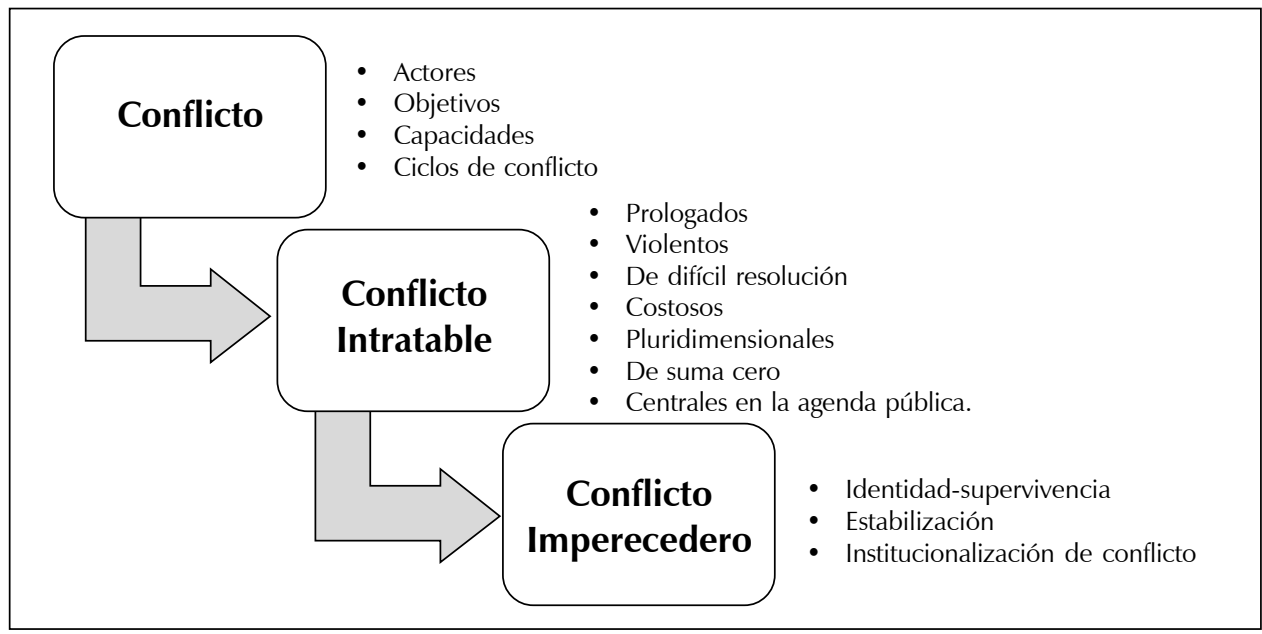

Gráfico 1.

Fuente: Diseño del autor. 


\section{TEORÍA GENERAL DE CONFLICTOS}

Como herramienta de acercamiento a los conflictos se plantean 4 preguntas fundamentales que servirán como mecanismo de acercamiento al modelo de análisis planteado en este artículo: ¿Qué son los conflictos? ¿Cómo son los conflictos? ¿Cómo clasificar los conflictos? ¿Cómo se desarrollan los conflictos?, a través de las cuales se establece una conexión directa con la naturaleza, elementos, morfología y procesos de los conflictos.

En la primera parte de la sección se encara la pregunta ¿Qué son los conflictos?, para luego abordar las restantes en la parte final de la sección.

\subsection{NATURALEZA DE LOS CONFLICTOS}

Remo Entelman (2002), en Teoría de los Conflictos: Hacia un Nuevo Paradigma, se propone desarrollar un mapa general de los conflictos, a partir de un ejercicio de ingeniería inversa que le permita extraer del amplio mundo de las especificidades provenientes del estudio de este fenómeno en áreas como el derecho, las relaciones internacionales o la violentologia, una descripción que "responda a las características genéricas de todos los tipos de enfrentamientos entre seres humanos, individuos o agrupados, que puedan describirse como subdivisión del amplio género conflictos" (Entelman, 2002, p. 44).

Con este propósito en mente, Entelman se lanza en búsqueda de una definición general para el universo total de los conflictos, para lo cual se vale de dos proposiciones fundamentales.

La primera proposición hace referencia a la necesidad de entender al conflicto como una relación social que se establece entre dos o más individuos, en la cual las agentes desarrollan acciones de carácter reciproco, por lo que cada uno de sus movimientos se explica en razón del vínculo que los une y la consideración del eventual comportamiento de su contraparte (Entelman, 2002:43-49).

Como segunda proposición establece, dentro del espectro del total de posibles relaciones sociales donde se presentan conductas reciprocas, los conflictos se caracterizan porque los objetivos que pretenden alcanzar las partes se comprende por ellas como incompatible o mutuamente excluyente, sea esta apreciación real o ficticia (Entelman, 2002:43-49).

A partir de estas dos proposiciones es posible plantear a los conflictos como una relación social en la que se involucran dos o más agentes, por medio de conductas reciprocas, bajo la presunción de los involucrados de estar persiguiendo metas u objetivos mutuamente incompatibles, dentro de un vínculo en el cual lo que obtiene una de las partes necesariamente redunda en una 
menor posibilidad de obtención o disfrute del elemento en disputa para su contraparte (Entelman, 2002:43-49).

Alcanzada esta perspectiva general de los conflictos, resulta clave reflexionar en torno al carácter complejo y dinámico del fenómeno, como epicentro de su desarrollo práctico y real.

Es en esta dirección que Josep Redorta (2004:46-48) invita a reconsiderar la forma en la que se han pensado los conflictos desde las ciencias sociales. El centro del cambio de perspectiva propuesto por Redorta se encuentra en la redefinición de las condiciones de funcionamiento, o dinámicas de movimiento, bajo las cuales se abordan los procesos sociales, pensándolos como realidades complejas que se ubica en el campo de lo no lineal y del pensamiento no determinista (Redorta, 2004:49-52).

El reto de pensar los conflictos en términos de la complejidad se encuentra en superar la búsqueda de variables o elementos aglutinaros o explicativos de la totalidad de las realidades conflictivas (Redorta (2004:69-70) ubica un total de 18 variables de importancia, a las que el analista deberá poner atención en su trabajo, sin que esto convierta en factor explicativo por valor propio a ninguna de estas dimensiones señaladas: metas, contexto, partes, poder, fuentes, patrón, función, expectativas, eje, vivencias, desencadenantes, ciclos, métodos, normas, complejidades, estilos, efectos, y ámbitos), para comprender las disputas humanas en términos de la relación que establecen múltiples variables que dan vida a sistemas dinámicos, en los cuales cada uno de los elementos se interrelaciona con los demás en procesos de interdependencia (Redorta, 2004).

Para comprender la necesidad de analizar los conflictos por las dinámicas de funcionamiento del sistema que componen sus variables, conviene llamar la atención sobre 3 corrientes de la complejidad, cuyos conceptos se hacen parte central del análisis de cualquier tipo de proceso social.

1.1.1. Lógica borrosa. Se funda en el manejo de la indeterminación, la ambivalencia y las zonas grises de los procesos. En este sentido, la borrosidad entiende la pertenencia o identidad de un fenómeno o cosa en términos de grado, y no de exclusión o inclusión. La realidad es imprecisa y no ideal, por lo que la parte pertenece al todo en gradualidades aceptables, y no en parámetros totales (Redorta, 2004:52-54).

1.1.2. Teoría de Catástrofe y autopoiesis. La idea del punto de catástrofe se refiere a los cambios y discontinuidades que se presentan en los fenómenos, cuando estos alcanzan puntos críticos que determinan las continuidades y las rupturas. La catástrofe, apoyándose en la utopoiesis, determina la tendencia de los sistemas a la organización en las formas que resultan más armónicas y estables. Estas nociones juegan con la idea del cambio y las continuidades de sistema, aceptando ambos aspectos de las realidades complejas (Redorta, 2004:54-59). 
1.1.3. Atractor y equilibro de sistema. El atractor se refiere a las tendencias de largo plazo de los procesos dinámicos, en las cuales es posible prever patrones estables y continuidades derivadas de situaciones o procesos continuos en el tiempo. En este sentido, el equilibrio de sistema proyecta que la complejidad tiende a encontrar un equilibrio sobre el que se desarrollan las interacciones, y se proyectan los cambios (Redorta, 2004: 57-58).

A lo que invitan estas nociones es a entender los procesos sociales como un todo, en el que la composición del sistema genera patrones identificables, sometidos al cambio constante por la dinámica propia de la interacción de sus variables, y no por relaciones simples de causa y efecto entre sus componentes.

\subsection{ESTRUCTURA DE CONFLICTO: MAPEANDO EL FENÓMENO}

Ahora que se ha abordado la naturaleza (¿qué son?), es momento de abordar las tres preguntas restantes formuladas al inicio de esta sección: ¿Cómo son los conflictos? ¿Cómo se clasifican los conflictos? ¿Cómo se desarrollan los conflictos?

La primera y segunda interrogante será desarrollada a continuación bajo la noción de morfología, desde la cual se perfilaran los componentes y taxonomía de conflictos. Por su parte, la última pregunta se encara al final de la sección, en la cual se describirán las dinámicas generales del proceso conflictual.

1.2.1 Morfología del conflicto. Como se estableció en la sección anterior, el entendimiento complejo de las dinámicas, formas y procesos conflictivos pasa por establecer un modelo de análisis que, en vez de tomar una variable como epicentro de clasificación, entienda a los conflictos a través de las diferentes formulaciones y patrones que adquieren las interacciones e interdependencias de sus componentes, como un sistema complejo y total.

Josep Redorta (2004), al abordar el concepto de morfología (como el estudio de la forma) de los conflictos, encara esta problemática dando una solución que satisface tanto las necesidades de la complejidad, como búsqueda de clasificaciones taxonómica eficientes y aplicables. La propuesta del autor se basa en pensar los conflictos por la forma que en la práctica adquiere cada proceso conflictivo, como totalidad sistémica, que permiten distinguir las características propias de una confrontación con respecto a otra (Redorta, 2004:97-108).

El autor propone determinar casos ideales, o moldes aplicables, que regidos por las nociones de la borrosidad y la psicología de las Gestalt', permitan pensar la identificación y clasificación de

1. La psicología de las Gestalt, que trabajando desde las la teoría de la percepción, proponía la ley de la pregnancia, como la cualidad de toda las organizaciones geométricas de adquirir la forma que le sea mejor, más sencilla y estable (Oviedo, 2004: 89-96). 
conflictos a partir de una estructura constante y reglas de juego reiteradas para cada tipo base de conflicto (Redorta, 2004:97-108).

Visto de esta forma, el perfil de cada molde ideal de conflicto necesita el establecimiento de un patrón de inclusión, que por oposición también se constituye como el rango de exclusión, que en cualquier caso se constituye como el referente invariable de pertenencia a cada categoría, que aunque de caso a caso puede presentar ligeras variaciones (puesto que ningún conflicto es exactamente igual al otro), es en sí mismo un rasgo elemental que diferencia a un tipo de conflicto de otro (Redorta, 2004:97-108).

1.2.2. Proceso o ciclos conflictuales. Los conflictos son procesos que cambian a lo largo del tiempo, en los que la hostilidad entre los actores varía durante largos periodos de tiempo. Al hablar del proceso conflictual, se está haciendo referencia entonces a la interacción entre dos elementos, tiempo e intensidad, dentro de las dinámicas de los ciclos del conflicto (Redorta, 2004:74-79).

En sí mismo determinar el punto de inicio de un conflicto es difícil, cuando no imposible, puesto que la contradicción de voluntades antes de hacerse manifiesta conlleva un largo periodo de latencia en el que las fuerzas van determinando la necesidad o no recurrir a la violencia. De igual forma, el momento de conclusión de un conflicto es en igual medida incierto, puesto que la inactividad o baja hostilidad por largos periodos de tiempo no significa necesariamente la extinción o terminación del marco general de una conflagración.

Sin embargo, el tiempo si se constituye como una de las variables fundamentales dentro de la compresión de los conflictos, puesto que su carácter prologado, olvidándose de los puntos de inicio y posible terminación, si determina el desarrollo o profundización de las dinámicas conflictivas, y la mayor o menor tendencia al uso de la violencia. El punto se encuentra en que el análisis del conflicto en el tiempo se debe realizar retomando la idea de morfología, en función de las formas que adquiere en el tiempo la interacción hostil entre las partes.

Desde esta perspectiva, el desarrollo de una disputa se basa en dos procesos inversos (escalda y desescalada), y dos etapas cumbre (estancamiento y punto de madurez), que se pueden presentar de forma cíclica a lo largo de los periodos de activación y latencia de las hostilidades (Redorta, 2004:74-79).

En primer lugar se tienen el concepto de escalada, como el proceso de expansión de las dinámicas conflictivas (violencia, espacios de combate, motivación grupal e implicación de terceros) entre los actores en disputa. Por su parte, las desescalada se define como el proceso contrario, en el que la hostilidad manifiesta una tendencia claramente regresiva (Redorta, 2004:74-76). 
Estas dos dinámicas interactúan a lo largo del tiempo, reduciendo o aumentando la intensidad del conflicto, dependiendo de los recursos disponibles, los costos asignados por las partes a aumentar o reducir la beligerancia, la efectividad de las estrategias implementadas y el estado de la legitimidad o soporte social a la continuación del conflicto (Redorta, 2004:77).

La interacción entre estos dos procesos permite la existencia de episodios de estancamiento, en los que las partes pierden interés en continuar con la competición, y se abren espacios de cooperación o entendimiento en temáticas diferentes a la generadora de la incompatibilidad de intereses. Estos periodos se suelen acompañar de desescalonamientos, que no implican la terminación del conflicto, puesto que el estancamiento se puede convertir en una oportunidad para recobrar capacidades y reactivar la confortación (Redorta, 2004:77).

A esta dinámica Redorta (2004) adiciona el punto culminante o de madurez del conflicto, como el momento en que las dinámicas conflictivas se empiezan a trasformar hacia espacios de menor confrontación y hostilidad. Este momento cumbre se suele alcanzar cuando se configura un escenario de empate entre las partes (ninguna en capaz de doblegar a la otra), se pierde control sobre el conflicto, la violencia alcanza un punto inaceptable o en los cálculos costo beneficio de los involucrados la cooperación se torna más atractiva (Redorta, 2004:77-78).

\section{DE LOS CONFLICTOS INTRATABLES}

Aunque ya se advirtió en la introducción, es clave recordar que lo que se está buscando en el presente artículo es la formación de una categoría aglutinadora para la comprensión del efecto del tiempo en los conflictos intratables, que se ha denominado aquí como lo imperecedero. En función de ello, se establecerá un molde ideal (en términos morfológicos) para la intratabilidad y lo imperecedero aplicando como regla de inclusión a la categoría tres factores centrales: primero, el involucramiento de la identidad grupal (objetivo de carácter trascendente) como factor de oposición de los agentes en conflicto. Segundo, la institucionalización de las dinámicas violentas como elemento de transición generacional. Tercero, el posicionamiento del conflicto como atractor de sistema para la estabilización de la conflagración.

En términos metodológicos, en la primera parte de la sección se dará desarrollo a la identidad y al colapso de la multidimensionalidad como factores centrales en la conformación de las lógicas morfológicas de la intratabilidad, para luego pasar en la última parte del artículo, al análisis pormenorizado de la formación de la propiedad de lo imperecedero, a través de la acción del tiempo sobre las dinámicas de la identidad de conflicto, la institucionalización y la estabilización de las lógicas de violencia de los conflictos intratables e imperecederos. 


\subsection{DE LA INTRATABILIDAD}

Ahondando en los rasgos centrales de los conflictos intratables, pero enfocándose en la forma en la que las dinámicas socio psicológicas impactan el carácter intratable de una confrontación, Daniel Bar-Tal (2007), en "Sociopsychological Foundations of intractable conflicts", define 7 factores centrales en la comprensión de la relación entre intratabilidad y desarrollo de la infraestructura psicosocial.

El primer elemento se refiere nuevamente al carácter prolongado de este tipo de conflictos (BarTal, 2007:1432). Al abarcar el espectro de una o varias generaciones, la conflagración constante se vuelve parte de la compresión de la realidad que desarrolla la totalidad de una comunidad, generando dinámicas de adaptación social y psicológica a todo el bagaje de sentimiento negativos y situaciones estresantes que se producen en un estado constante se sosiego e incertidumbre originado en la disputa violenta.

Bar-Tal reconoce, como segundo factor, que los niveles de violencia que rodean a un conflicto terminan por marcar de forma radical la emocionalidad social, y por esta vía, la capacidad de los individuos y el grupo de interpretar y explicar la realidad (Bar-Tal, 2007:1432).

El tercer rasgo fundamental es la inexistencia de una posibilidad, detectable por los involucrados (tanto combatientes como no combatientes), de una vía de resolución del conflicto (Bar-Tal, 2007: 1432). En esto es clave identificar que la solución no se presenta en términos pacíficos ni violentos, porque ninguna de las partes es capaz de doblegar a la otra por la fuerza, y de igual forma, de las experiencias de la sociedad con respecto a experimentos de resolución pacífica, si existen, los mecanismos han carecido de funcionalidad y eficacia en la mitigación del conflicto.

Como cuarto y quinto elemento se destaca, dadas las lógicas extensivas de la intratabilidad, que este tipo de conflictos, en primer lugar, demandan una el involucramiento social de la mayoría de los recursos producidos por la totalidad del grupo. En segundo lugar, el conflicto adquiere un carácter pluridimensional, que involucra múltiples aspectos de la vida comunitaria bajo lógicas de retroalimentación positiva, que tienden a impulsar la violencia (Bar-Tal, 2007: 1432-1433).

Como sexto factor, el conflicto que adquiere una dinámica de intratabilidad se convierte con el tiempo a las lógicas de los juegos de suma cero (Bar-Tal, 2007: 1433). En lo que respecta a la percepción que los involucrados, al estar en juego relaciones de identidad y supervivencia, el conflicto adquiere una lógica de costo-beneficio en la que la pérdida de cualquier elemento por el bando propio, es una amenaza directa a la existencia misma del grupo, en beneficio del contradictor amenazante.

Como elemento final, al convertirse el conflicto en el factor central del desarrollo y definición de la vida e interrelación social, la agenda pública (tanto la que se desarrolla por los medios de 
comunicación, los principales líderes de opinión, instituciones y políticos, como por las personas en las discusiones comunes) se ve absolutamente copada por los elementos derivados de la confrontación (Bar-Tal, 2007: 1433).

Estas 7 lógicas de funcionamiento de la intratabilidad desarrollan un imaginario que aúna de forma directa las condiciones y lógicas de confrontación, con la formación de la identidad colectiva y supervivencia grupal.

\subsection{LA IDENTIDAD COMO FACTOR DE CONFLICTO}

En la conceptualización propuesta por Daniel Bar-Tal (2007) se pueden identificar las dinámicas de la intratabilidad a partir de la interacción entre el tiempo de confrontación, la trascendencia de los objetivos en disputa, la identidad grupal y la supervivencia.

El autor establece la conexión entre estos factores a partir de la forma en que se desarrolla la infraestructura psicosocial, como base de la conformación de la identidad grupal, en un contexto de conflicto intratable.

En términos generales, la infraestructura psicosocial se podría entender como el conjunto de los saberes comunes que ayudan a las personas a operar en su vida diaria. Son las creencias sociales, colectivamente compartidas, sobre los procesos y elementos de interés prioritario para la sociedad (Bar-Tal, 2007:1435).

De esta forma, a partir de las características que la infraestructura psicosocial toma en cada grupo, las sociedades y los individuos que las componen son capaces de entender su papel en el mundo, el lugar, funciones y patrones de relación que sus unidades desarrollan dentro del contexto global del grupo y para con otras colectividades (interacción e interrelación inter e intra grupal). Los grupos destacan de sí mismos ciertas características en vez de otras, recuerdan de forma particular su pasado, entienden su presente y proyectan su futuro. En otras palabras, la infraestructura psicosocial es el insumo elemental con el que se compone la identidad grupal. El elemental pero trascendental ¿Quiénes somos? ¿Qué queremos? ¿Hacia dónde vamos? ¿Por qué lo hacemos?

En este sentido, la infraestructura psicosocial se alimenta de las particulares necesidades que cada grupo desarrolla con respecto al contexto al que se enfrenta, y lo transmite a sus unidades a través de los principales medios de comunicación y mecanismos de aprendizaje social (colegios, los medios de comunicación, los políticos, las producciones culturales, etc.) (Bar-Tal, 2007:1444-1445). 
Dicho esto, el trabajo emprendido por Bar-Tal adquiere su dimensión fundamental cuando el autor propone el análisis de las particulares condiciones bajo las que se desarrolla la infraestructura psicosocial en condiciones de conflicto.

Para Bar-Tal (2007:1434-1435) es claro que las sociedades que experimentan largos y virulentos procesos conflictuales, deben desarrollar mecanismo que les permitan vivir en medio de altos niveles de tensión social y emocionalidad negativa, derivados de la violencia como factor central en el desarrollo colectivo. Para lograr esto, el conjunto social se ve obligado a generar una serie de herramientas que cubran las necesidades psicosociales de la población. Este proceso determina para estas sociedades una particular compresión de la realidad que se configura teniendo al conflicto como epicentro único.

Estos instrumentos autogenerados se encaminan a garantizar altos niveles de resiliencia a la emocionalidad negativa, proveyendo a los miembros de una sociedad de parámetros de identidad positiva, ciertos niveles de seguridad y de una información que se considera como socialmente relevante al respecto del entorno inmediatito (Bar-Tal, 2007:1441-1443).

En todos los casos, la infraestructura psicosocial trabaja en pro de una noción de constante sacrificio individual por el bienestar del colectivo, como razón explicativa al contante flujo de violencia en el entorno, que permite a las personas desarrollar una perspectiva de optimismo sobre una posible victoria (o cuando menos la lejanía de la derrota) que de sentido a la continuación de la lucha (Bar-Tal, 2007:1441-1443).

Bajo esta dinámica, la sociedad se mantiene preparada para afrontar situación de tensión y violencia prologada, legitimando, a través del otorgamiento de sentido al conflicto, la violencia ejercida por el propio grupo y deslegitimando al otro en su causa y en su identidad y condición humana. Este último factor, la deshumanización, se vuelve central dentro de las lógicas de los conflictos prolongados en los que la violencia se ejerce en manera descontrolada y extensible, por periodo de escalonamiento y alta agresividad.

Bar-Tal (2007:1436-1440) identifica la memoria colectiva, el ethos del conflicto y la orientación emocional colectiva como las dimensiones desde los cuales es posible entender el funcionamiento de la infraestructura psicosocial en tiempo de alta agresividad y violencia prolongada.

En términos generales, la memoria colectiva hace referencia a las narrativas (saberes colectivos) que desarrollan las sociedades para explicar el estado actual de su contexto, basándose en la reconstrucción de acontecimientos y procesos de alto impacto (Bar-Tal, 2007:1436-1438). Es clave reconocer que la memoria colectiva no es una reconstrucción objetiva, aunque para el cuerpo social se entienda como tal, de los acontecimientos sucedidos, sino una selección de acontecimientos rememorados con altas dosis de emocionalidad, y que responden a las necesidades grupales a lo largo del tiempo. 
En situaciones de conflicto la memoria se convierte en un justificante sobre el inicio conflicto y el desarrollo de la confrontación, a partir del cual se deslegitiman los reclamos del oponente y se crea una imagen positiva propia por medio de la victimización frente al agresor externo.

Mientras la memoria colectiva reconstruye los hechos puntuales del proceso conflictivo, el ethos se enfoca en la generación de un marco discursivo que de sentido al ipor qué? de esta situación (Bar-Tal, 2007:1438-1439). El ethos debe generar un vínculo directo entre el estado actual de las cosas, y un futuro idealizado y victorioso que legitime las metas grupales (visión de la paz futura), genere cohesión interna (patriotismo o unidad), estigmatice al oponente y generando niveles mínimos de seguridad psicosocial en los miembros del grupo.

Las tendencias emocionales colectivas son vistas por Bar-tal como la predisposición de los grupos humanos a experimentar ciertos ambientes emocionales, derivados del entorno transicional y cultural de cada grupo humano (Bar-Tal; Halperin y De Rivera, 2007:443-455). Visto de esta forma, el contexto cultural define las formas socialmente aceptadas de expresar la emotividad y los sentimientos, al mismo tiempo que condiciona o predispone a los grupos a experimentar un tipo de emociones en particular. A su vez, el contexto emocional transicional se refiere a las perturbaciones de la atmosfera emocional derivadas de procesos y acontecimiento de alto impacto psicosocial en los grupos.

Para Bar-tal (2007:1439-1440), en contexto de conflicto intratable las sociedades se configuran bajo dos sentimientos negativos centrales: el miedo y el odio. A través de la combinación de estas dos emocionalidades se prepara a la sociedad para dar respuestas adaptativas frente al peligro, con el fin de maximizar la supervivencia. De igual forma, se crea la imagen de un enemigo sobre el cual hacer recaer la responsabilidad por la agresión sufrida en el tiempo.

Las dinámicas de configuración de estos tres componentes psicosociales forman la identidad grupal de una sociedad, que se define a sí misma en función del conflicto en el que vive.

Esta dinámica que vincula al conflicto y a la identidad explica porque Bar-Tal (2007:1433) advierte que para los participantes de un conflicto intratable la disputa se convierte en un juego de suma cero. Si una larga narrativa de lucha, con la emocionalidad negativa de corto (coyuntura) y largo (cultura) plazo que esta genera, se convierten en el insumo básico a través del cual un grupo es capaz de entender su realidad y el lugar que ocupa en el mundo como colectividad, la pérdida del mismo se convierte en la diferencia entre existir o no, mientras que el derrotar al contrario (completamente deshumanizado) se trasforma en el edén donde se proyectan los objetivos y ambiciones a cualquier escala.

Este parámetro, conflicto-identidad-supervivencia-, determina la noción base de un valor de naturaleza trascendental como epicentro de la disputa. Dentro de la identidad grupal de ambos bandos, no se pueden entender a si mismo por fuera de la conflagración, ni se puede imaginar en un escenario que no sea la derrota del contrario, en otras palabras, un juego de suma cero. 


\subsection{MORFOLOGÍA DE LA INTRATABILIDAD}

Es menester advertir desde el inicio, para la compresión de la morfología de la intratabilidad, que ningún conflicto es intratable en su génesis (Gray; Coleman y Putman, 2007:1416).

La perdurabilidad, hostilidad excesiva y la resistencia a la transformación positiva o pacifica, como características preponderantes de la intratabilidad, son el resultado de periodos de incremento descontrolado de la hostilidad y violencia relacionada con el conflicto (Coleman; Vallacher; Nowak y Bui-Wrzosinska, 2007:1457-1463). Visto desde las apreciaciones hechas en la sección anterior, en las que se delimito la identidad como patrón de inclusión dentro de la morfología de la intratabilidad, a continuación se definirán las lógicas de inclusión para las dos condiciones restantes: intensidad y tiempo, que como se explicó antes, configuran las lógicas de comprensión de los ciclos conflictuales.

Bajo esta perspectiva, la aparición de la intratabilidad deviene de los ciclos de escalonamiento y desescalonamiento que se producen alrededor de los conflictos, que son a su vez el producto de la interacción dinámica de las múltiples variables que afectan el desarrollo de un proceso conflictivo.

Visto de esta forma, la determinación de la morfología de la intratabilidad demanda poner atención a las dinámicas de desarrollo de los ciclos conflictuales, en tanto patrón de advenimiento de los momentos de escalonamiento y desescalonamiento del conflicto, y a las forma en que se generan las lógicas de perdurabilidad y transito generacional, como determinantes del tiempo como factor central de la intratabilidad.

A las condiciones de perdurabilidad (trasfondo imperecedero y de retroalimentación de violencia) se le dedica la sección siguiente, por lo que en esta la explicación se enfoca el escalonamiento como condición de sostenimiento y creación de la intratabilidad.

El enfoque de Coleman, Vallacher, Nowak y Bui-wrzosinska (2007), en "Intractable Conflict as an attractor: A Dynamical systems approach to conflict escalation an intractability", resulta absolutamente esclarecedor sobre la relación entre los ciclos de hostilidad y el desarrollo de la intratabilidad.

Estos autores invitan a pensar los conflictos intratables como la interacción dinámica de múltiples elementos que definen la relación entre los patrones psicológicos internos de las personas, y su forma de relación inter e intra grupal (Coleman; Vallacher; Nowak y Bui-Wrzosinska, 2007:1457-1463). La propuesta de Coleman, Vallacher, Nowak y Bui-wrzosinska aplica la lógica sistémica al entender que las dinámicas de interdependencia que afectan a las variables del conflicto, se modifican entre sí a través de procesos de retroalimentación positiva o negativa, según la cual la activación de una variable de conflicto empuja a las demás en la misma tendencia. 
Para Coleman, Vallacher, Nowak y Bui-wrzosinska (2007:1457-1463) esta dinámica de retroalimentación se observa desde la interacción que va desde las fuerzas promotoras del conflicto (intereses, agravantes, etc.), y la intensidad con la que se desarrolla la confrontación. Desde esta conceptualización, entre mayor sea el grado de separación o proximidad entre los dos tipos de variables, mayor será el grado en que la actuación particular de los bandos tiene una influencia directa en la hostilidad propia del conflicto.

El sistema adquiere entonces su carácter intratable cuando la distancia entre las fuerzas promotoras del conflicto y la intensidad del mismo alcanza un punto catastrófico de cambio, determinado por el nivel de violencia ejercido por las partes, en el que en la práctica los diferentes elementos del sistema conflictivo se fusionan, y las variable se independiza de la voluntad y acción de los actores y factores promotores del conflicto, convirtiendo a la violencia en una dinámica impulsada y retroalimentada por las condiciones del contexto, extensible a la totalidad de los conjuntos sociales, sin el control centralizado de los liderazgos intra grupales (Coleman; Vallacher; Nowak y Bui-Wrzosinska, 2007:1460).

En este estado las dinámicas de violencia intra e inter grupales adquieren un movimiento propio, independiente de la hostilidad que se demuestren ente si las partes, bajo una reproducción sistemática de la violencia en la cual se produce lo que Coleman, Vallacher, Nowak y Bui-wrzosinska (2007:1463-1467) denominan colapso de la multidimensionalidad de las relaciones sociales.

Este fenómeno es entendido por los autores como la reducción, a una estructura simple de relación directa, de la multiplicidad de campos y espacios en los cuales se desarrollan las interacciones y relaciones humanas (Coleman; Vallacher; Nowak y Bui-Wrzosinska, 2007:1463-1467).

En este sentido, la violencia alcanza un nivel límite en el que la retroalimentación positiva de la hostilidad se vuelve multidimensional y casi directa, promoviendo el escalonamiento constante de la hostilidad al interior del sistema, sin importar cuál de las variables sea estimulada. Un conflicto en cualquier área (deportiva, económica, etc.) desencadena una escalada de episodios violentos entre las partes.

El punto se encuentra en que la fusión o colapso de la multidimensionalidad permite, por un lado, que cualquier interacción en cualquier espacio que se considere negativa, estimule a todo el sistema a aumentar el nivel de beligerancia general, y por otro lado, extingue la posibilidad de establecer patrones de desescalonamiento por medio de la creación de entornos de relación basado en la cooperación intergrupal en procesos no vinculados de forma directa con el conflicto (Coleman; Vallacher; Nowak y Bui-Wrzosinska, 2007:1463-1467).

A partir de este proceso se genera un distanciamiento absoluto de los agentes, que imposibilita el desarrollo de espacios de interacción no conflictiva, mientras que se permite un vínculo directo de ellos a través de la confrontación y la violencia derivada de las acciones intergrupales hostiles. 


\section{DE LO IMPERECEDERO: INSTITUCIONALIZACIÓN DE LAS DINÁMICAS DE CONFLICTO Y ATRACTORES DE SISTEMA}

Como se mencionó en la sección anterior, una de las características fundamentales de los conflictos intratables es su carácter prolongado en el tiempo. Las complejidades que esta dimensión encierra, como variables de análisis de los conflictos intratables, es en lo que se concentra la presente sección, y lo que inspira la realización de este artículo.

Y es que al considerar con detenimiento las dinámicas de funcionamiento de estas disputas, se configura una lógica aparentemente contra intuitiva que desafía, o como mínimo cuestiona, el funcionamiento lineal (causa y efecto) en el que se han basado las explicaciones tradicionales de los conflicto.

En este sentido, se vuelve necesario considerar lo siguiente: en un conflicto intratable, a pesar de las elevadas dosis de violencia y energía (recursos, población, atención social, etc.) que las sociedades deben destinar al sostenimiento de los procesos conflictivos, con el pasar del tiempo se aumenta la disposición para luchas y la capacidad para destinar la totalidad de los recursos a la perpetuación, o incluso expansión, de las dinámicas de conflictividad a gran escala.

En un conflicto intratable, a diferencia de lo que indicaría cualquier aproximación convencional, el paso del tiempo no implica el agotamiento de los recursos disponibles ni tampoco conlleva a la disposición de los bandos a buscar soluciones alternativas a la violencia, sino que en realidad remarca y predispone a las generaciones de recambio a la violencia, y a la búsqueda de nuevas formas de captura de recursos para sostener la guerra.

Aquí se configura una dinámica que supera con plenitud la consideración de lo simplemente prologado, como aquello que perdura por un periodo considerable, por una interpretación en la que el tiempo actúa en una dimensión diferente, puesto que el mismo se convierte en un factor de refuerzo de la conflictividad grupal.

En este artículo, como propuesta de una nueva categoría de trabajo y análisis, se propone entender lo imperecedero, dentro del marco de la intratabilidad, como una propiedad por medio de la cual las dinámicas de confrontación son capaces de perpetuarse por tiempo indefinido, a través del sostenimiento de sus lógicas de funcionamiento mediante la expansión y adaptación de sus características al cambio de contexto y a los tránsitos generacionales.

Es clave identificar que lo imperecedero como categoría de análisis no crea una nueva tipología separada de los ya conceptualizados conflictos intratables, aquí lo que se desea complejizar y problematizar el entendimiento del tiempo como variables central de la intratabilidad, redimensionándolo a través de la idea de lo imperecedero. 
Bajo esta propuesta, la aparición de un conflicto intratable e imperecedero depende de una compleja pero sostenida interacción entre el tiempo (visto desde los ciclos de escalonamiento del conflicto) y la consolidación de una identidad grupal (a través de la infraestructura psicosocial) que responda de forma exclusiva a la perpetuación de las dinámicas de violencia intergrupales. Los mecanismos que aseguran la perdurabilidad de los conflictos intratables e imperecederos se puede expresar a través de dos procesos de lógicas coincidentes, que se retroalimentan de forma positiva a través del tiempo: la estabilización (sostenibilidad de la violencia) e institucionalización de conflictos (perdurabilidad de la agresión).

La articulación de estas dos dinámicas va a permitir asegurar un sistema a largo plazo de retroalimentación generacional de la identidad de conflicto, por medio de la alimentación constante de las narrativas de lucha y la emocionalidad negativa que componen la infraestructura psicosocial, además de la creación de condiciones y escenarios escalonados de confrontación en múltiples niveles y áreas, cada vez más diversificadas con respecto al elemento original, o factor desencadenante de la conflagración.

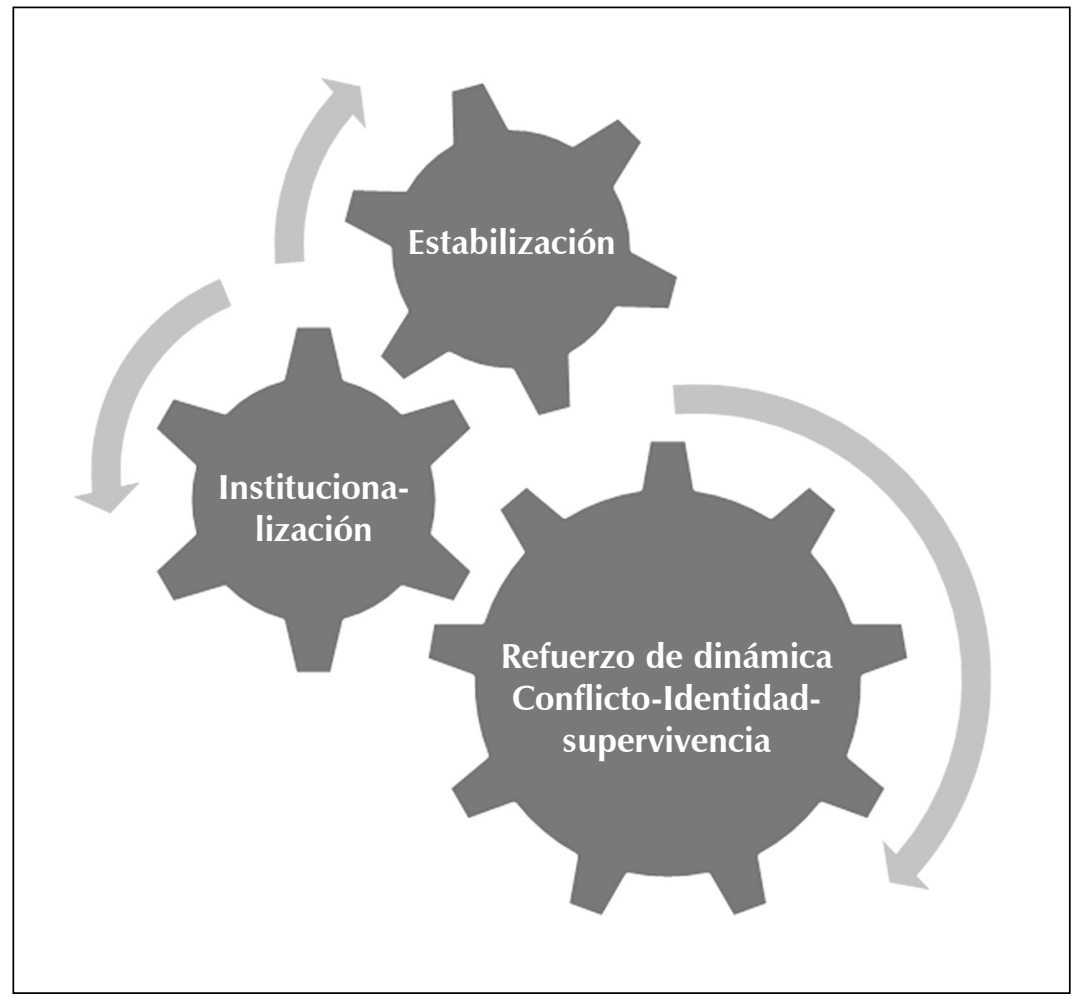

Grafico 2: Sistema de institucionalización y estabilización Fuente: Diseño autor, basado en (Coleman; Vallacher; Nowak y BuiWrzosinska, 2007; Gray; Coleman y Putman, 2007 ; Bar-Tal, 2007) 
A continuación se describirán los mecanismos de estabilización e institucionalización, para terminar con la caracterización de la configuración de la identidad grupal en los conflictos intratables e imperecederos.

\subsection{INSTITUCIONALIZACIÓN DE CONFLICTOS}

En cuanto al proceso de institucionalización, Barbara Gray, Peter Coleman y Linda Putman (2007:1423-1424) señalan que a través del escalonamiento de la violencia van apareciendo patrones regulares de comportamiento dentro de los conjuntos sociales, que son constantemente reforzados por el consenso social resultado de la medicación cognoscitiva de la infraestructura psicosocial, que va generando los hábitos de acción y las herramientas emocionales necesarias para que las personas vivan en medio de la violencia.

Esta normativización de la vida en conflicto implica la regulación del comportamiento individual, intra e inter grupal, a través de mecanismos de sanción social a los patrones de acción desviados, el desarrollo de expectativas con respecto a cómo se comportaran los miembros del propio grupo y los rivales y la aparición de secuencias y roles rutinarios (culturalmente aceptados) derivados de la asimilación del conflicto como factor clave en el subconsciente individual y grupal (Gray; Coleman y Putman, 2007:1420-1422).

La institucionalización presenta consecuencias positivas y negativas. Por un lado, permite mantener a los grupos cohesionados durante largos periodos de tensión social, y por el otro, dificulta la resolución de conflictos por la regularización, estandarización y refuerzo de las dinámicas de violencia dentro de los grupos.

Lograr el transito generacional de los patrones regulares de comportamiento violento, factor que le da el carácter imperecedero a un conflicto, demanda la reproducción de las dinámicas de conflicto a través de su presencia contante en los medios de comunicación y espacios de educación social.

Al respecto de esto, en "Collective Emotions in Conflict Situations: Societal Implications.", BarTal, Halperin y De Rivera (2007:443-455) proponen que el principal mecanismo de expresión o materialización de los climas emocionales que se configuran al interior de un grupo, son los medios culturales de comunicación (libros, películas, música, prensa, etc.). En ellos se expresan de forma prioritaria los sentimientos y emociones que se derivan de los procesos coyunturales y culturales que viven los seres humanos.

Bajo este parámetro, en el caso de las sociedades que se desarrollan en medio de un conflicto intratable, esos productos culturales serán la materialización y expansión del miedo y el odio como productos principales de los climas emocionales que se generan en medio de 
la hostilidad. La reproducción material de la memoria y el ethos de conflicto se convierte en el punto central de la agenda pública, copando también los materiales de aprendizaje y educación a los que se ven expuestos los niños y jóvenes del grupo.

Esta versión oficial de la historia, no solo es sostenida por la retroalimentación de los productos sociales y culturales, sino que también se convierte en fuente de legitimación política, por lo que es protegida, engrandecida y ritualizada por el aparato político gubernamental (Bar-Tal, 2007:1445).

En este sentido, toda la producción cultural de un grupo se va a ver atravesada por las lógicas que explican la raíz y estado actual del conflicto, bajo parámetros que validan la causa y violencia ejercida por el grupo propio, e invalidad y deshumanizan al contrario como epicentro de la emocionalidad negativa.

El punto se encuentra en que las herramientas de expresión culturas se convierten en la principal fuente de transmisión de información entre generaciones, revalidado y retroalimentado los patrones de vida que se establecen en condiciones de conflicto, en un nuevo grupo de personas que reproducirán las lógicas de acción durante otro periodo generacional. El transito temporal resulta especialmente significativo en el sentido en el que los procesos de socialización y rituales de ingreso a la vida grupal, de los niños y jóvenes de una comunidad, son atravesados e involucran de forma directa dinámicas relacionadas con el conflicto. En este sentido, toda la perspectiva y formación cognoscitiva de la generación de recambio se forma y adapta para repetir y reforzar las dinámicas conflictivas.

\subsection{ESTABILIZACIÓN DE LAS DINÁMICAS VIOLENTAS}

Para prolongarse indefinidamente, la intratabilidad depende de que en los periodos de estancamiento y desescalonamiento, donde se abren espacios de interacción positiva y cooperación mínima, sean utilizados por las partes para la retoma de fuerzas y el reavivamiento de capacidades que se enfocan en realimentar el conflicto, hacia una nueva temporada de escalonamiento.

Lo anterior se basa, en primer lugar, en que al alcanzar el colapso de la multidimensional los procesos de interacción positiva se ven imposibilitados por la unificación de las dimensiones sociales sobre el conflicto, convirtiendo cualquier tipo de intercambio en un terreno de competición y posible desarrollo de violencia.

En segundo lugar, el que los espacios de estancamiento funciones como repotencializadores del conflicto se explica a través de lo que Coleman, Vallacher, Nowak y Bui-wrzosinska (2007:1457-1462) denominan el conflicto como atractor de sistema. 
La perspectiva analítica utilizada por estos autores parte de la idea de que los sistemas complejos y dinámicos, como los conflictos intratables, a pesar de su predisposición al cambio tienden a constituirse bajo patrones estables de desenvolvimiento en el tiempo. Dentro de esta lógica, la noción de atractor, como fue explicada anteriormente, se entiende como una predisposición o punto homogeneizador de las relaciones dentro del sistema, que para los conflictos intratables e imperecederos se ubica en la reproducción de las dinámicas de violencia y conflicto Coleman, Vallacher, Nowak y Bui-wrzosinska, 2007:1457-1462; Redorta, 2004:57-58).

En términos sencillos, la idea del conflicto como atractor de sistema apunta a conciliar las nociones de cambio dinámico -representada en los ciclos de mayor o menos hostilidad-, los puntos de estancamiento -que se presentan de forma alternada a lo largo del proceso conflictivo- y el principio de equilibrio de sistema -que sugiere que sin importar un número significativo de alteraciones que se pueden experimentar en un proceso conflictual, este sostienen una tendencia irrestricta a volver a un cierto punto en el que el balance de fuerzas del sistema se equilibra-.

En sí misma la idea de atractor aplica la noción de institucionalización, puesto que la implementación de patrones normados y regularizados de comportamiento permite que el punto de equilibrio de sistema se encuentre en el conflicto, debido a que los periodos de calma o latencia se constituyen como acumuladores que desbalancean las cargas de las relaciones entre los agentes, para retornar a la hostilidad que rebalancea el sistema.

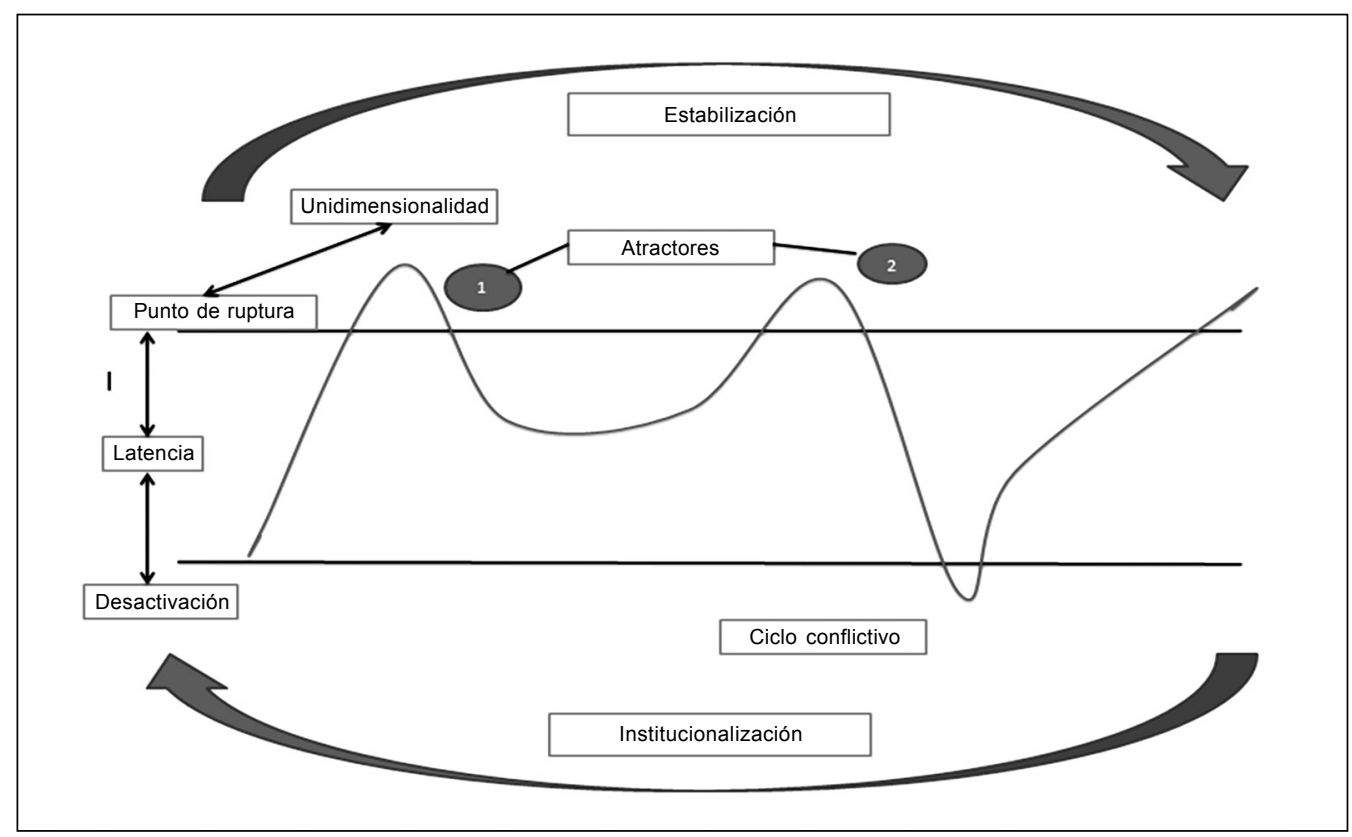

Grafica 3. Morfología de la Intratabilidad y lo imperecedero.

Fuente: Diseño del autor basado en (Coleman; Vallacher; Nowak y Bui-Wrzosinska, 2007; Gray; Coleman y Putman, 2007; Bar-Tal, 2007) 
Lo que resulta significativo es percibir los periodos de estancamiento, los desescalonamientos e incluso los puntos de madurez, que desactivan la hostilidad y pueden reconducir al sistema a un periodo de latencia, como la separación más drástica que enfrenta un sistema conflictivo intratable e imperecedero (punto ideal de intervención para la mediación y la construcción de alternativas de paz) antes de retornar, a través de la acumulación de emocionalidad negativa permitida por el colapso de la multidimensionalidad, al conflicto como punto atractor de sistema.

\subsection{IDENTIDAD GRUPAL EN LOS CONFLICTOS INTRATABLES E IMPERECEDEROS}

En este punto se ha hecho claro que a través de la institucionalización se asegura un sistema de retroalimentación y transito generacional, capaz de llevar las narrativas de conflicto a través del tiempo, reforzando el carácter negativo de su composición.

En esta misma dirección, al identificar al conflicto como punto atractor de sistema, se delimito la forma en la que los ciclos de escalonamiento y desescalonamiento crean los espacios a través de los cuales los bandos en conflicto se aseguran la capacidad de alimentar la hostilidad de manera constante en el tiempo.

Estas dos dinámicas aseguran, en términos materiales, la retroalimentación y el transito generacional del conflicto, para que el mismo pueda alcanzar la propiedad de imperecedero.

En lo que se refiere a la identidad grupal de conflicto, la dimensión imperecedera se va a caracterizar por una absoluta desconexión entre los objetivos trascendentales de cada bando, con realidades alcanzables dentro de un plano temporal ubicable en la realidad. En los conflictos intratables e imperecederos los objetivos de los bandos, y las narraciones que dan lugar y justificación a los mismos, pierden conexión con lo que se podría considerar como posible, o incluso imaginable, dentro de un rango de posibilidad negociable entre las partes. Se construyen objetivos en planos atemporales, que sostienen un sistema donde cualquier nivel de concesión o derrota dentro del conflicto es una amenaza a la supervivencia, $y$ la victoria resulta simplemente imposible en las condiciones narrativas en las que está planteada.

Gracias a este proceso de consolidación y refuerzo temporal de largo plazo, las narrativas que dan justificación y significado al conflicto (tanto en lo que se refiere a la memoria como al ethos del conflicto), se hacen cada vez más inexactas, desprendiéndose de manera drástica de los hechos en función de las necesidades grupales, que son constantemente cargadas de nuevos componentes de valor emocional negativo.

Esta lógica de funcionamiento permite que la identidad grupal vinculada a las narrativas de lucha, que configuran el entendimiento de la realidad de un grupo, adquieran dimensiones atemporales y ubiquen sus objetivos trascendentales en escenarios míticos. 
Dentro de este escenario se pueden identificar nociones de lucha religiosas de consolidación global, conceptos acerca de pueblos elegidos o destinados hacia un propósito fundamental, y proyectos nacionales o sociales con perspectiva universalista.

Al configurarse un estado de cosas como este, en el que ningún tipo de respuesta práctica puede dar cabida a un marco de mitigación o resolución de la hostilidad, el carácter imperecedero de un conflicto alcanza su dimensión plena, puesto que el mismo se asienta en una lógica autocontenida y de reproducción sistemática que confluye en lo indefinido o atemporal.

\section{CONCLUSIONES}

En las anteriores páginas se propuso una revisión de la idea de los conflictos intratables, poniendo el énfasis explicativo no solo a su desarrollo como fenómeno social, sino también en su ubicación dentro del campo de la teoría de conflictos.

El punto fundamental se encontró en problematizar lo que hasta ahora se ha entendido como el carácter prolongado de este tipo de conflictos. De esta forma se ubicó una importante falacia en las conceptualizaciones existentes en torno a esta variable de la conflictividad, puesto que el transito generacional y la plena disposición de lucha se basan en dinámicas de retroalimentación temporal que desafían la compresión tradicional de los conflictos, a través de los modelos lineales de causa y efecto.

Por esta vía se identificó que la alta hostilidad, el carácter prologado y la resistencia al cambio pacífico se ubican en la interacción entre los objetivos de carácter trascendente de los conflicto (la identificación identidad y supervivencia), y la composición de lógicas morfológicas que permitan el transito generacional y la estabilización de las dinámicas conflictivas.

Estos elementos permiten el refuerzo de las lógicas de funcionamiento de la identidad como factor de lucha dentro de los conflictos intratables e imperecederos, bajo un trasfondo que mantiene la lógica de juego de suma cero entre los bandos en disputa.

Hacia el final del texto se prestó atención pormenorizada a las lógicas de reproducción y cambio generacional que mantienen funcionando el conflicto durante periodos prologados de tiempo, atribuyendo a las ideas de estabilización, como punto de retorno del sistema hacia el conflicto, e institucionalización, como normalización y sostenimiento de patrones de violencia, un carácter explicativo dentro de la noción de lo imperecedero, como concepto de análisis de la variable tiempo dentro de la intratabilidad.

En cuanto al campo metodológico, se propuso la necesidad urgente de implementar explicaciones que encaren la lógica compleja de los conflictos, parándose sobre el dominio de las 
dinámicas de los conjuntos borrosos, los atractores y el equilibrio de sistema, como herramientas para sortear la complejidad, en vez de optar por modelos de causa y efecto que se quedan pobres en la compresión de la realidad.

De igual forma, la revisión se inspiró en la necesidad de crear modelos de análisis explicativo, que no solo se ubiquen dentro de la lógica de los manuales de intervención y resolución de conflictos, normalmente inútiles cuando se aplican en escenarios que cuentan con las complejidades expuestas anteriormente.

Es en este sentido que el material bibliográfico existente resulta insuficiente, puesto que el método de trabajo consolidado dentro del campo es partir de la necesidad inmediata de crear marcos universales para la resolución de conflictos, que al ser efectivos en algunos escenarios, se pretenden universalizar para todo contexto y campo.

En esta dirección, la propuesta metodológica que aquí se implementó, trata de partir del entendimiento profundo de los conflictos y no de su resolución universal. Bajo esta lógica se pretende subsanar la actitud reacia, en especial dentro de la academia latinoamericana, a la búsqueda por explicar la naturaleza básica de los conflictos, antes de aventurarse a plantear escenarios de resolución o manejo.

En todo caso, la intensión de este documento es abrir espacios y nuevas formas de pensar el material existente, dentro de lógicas de análisis diferentes. Lo aquí consignado no representa de ningún modo la última palabra en la materia, pero si una duda de fondo en torno a la manera como deseamos pensar los conflictos a cualquier escala.

\section{BIBLIOGRAFÍA}

- $\quad$ Bar-Tal, D. (2007). "Sociopsychological Foundations of Intractable Conflicts." American Behavioral Scientist. 50, 11, 1430-1453.

- $\quad$ Bar-Tal, D; Halperin, E. y De Rivera, J. (2007). "Collective Emotions in Conflict Situations: Societal Implications." Journal Of Social Issues. 63, 2, 441-460.

- Coleman, P; Vallacher, R; Nowak, A. y Bui-Wrzosinska, L. (2007). "Intractable conflict as an attractor: a dynamical systems approach to conflict escalation and intractability." American Behavioral Scientist. 50, 11, 1454-1475.

- Entelman, R. (2002). Teoría de los Conflictos: Hacia un Nuevo Paradigma. Barcelona, Editorial Gedisa. 
- Fernández, F. (2011). La ecuación de la Guerra. Málaga, Ediciones de Intervención Cultura Montesinos.

- $\quad$ Fisas, V. (2002). Cultura de paz y gestión de conflictos, Barcelona, Ediciones Icaria.

- Gray, B; Coleman, P. y Putman, L. (2007) "Introduction: intractable conflict: new perspectives in the causes and conditions for change." American Behavioral Scientist. 50, $11,1415-1429$.

- Joas, H. (2005). Guerras y modernidad. Estudios sobre la historia de la violencia en el siglo $X X$. Barcelona, Editorial Paidós.

- Oviedo, G. (2004) “La definición del concepto de percepción en psicología con base en la teoría Gestalt." Revista de Estudios Sociales. 18, 89-96.

- Pizarroso, A. (2005) Nuevas Guerras, vieja propaganda: de Vietnam a Irak. Madrid, Ediciones Catedra.

- Redorta, J. (2004) Cómo analizar los conflictos. La tipología de conflictos como herramienta de mediación. Barcelona, Editorial Paídós. 